\title{
Infliximab treatment in a paediatric patient with ulcerative colitis, who developed acute pancreatitis due to azathioprine during follow-up
}

\author{
Ahmet Basturk, Aygen Yilmaz, Meryem Keceli, Reha Artan \\ Department of Paediatric Gastroenterology, Faculty of Medicine, Akdeniz University, Antalya, Turkey
}

Gastroenterology Rev 2017; 12 (3): 235-237

DOI: https://doi.org/10.5114/pg.2017.70479

Address for correspondence: Ahmet Basturk PhD, Department of Paediatric Gastroenterology, Faculty of Medicine, Akdeniz University,
Akkuyu mh. 1039. sk. Onursal kop. G Blok No: 16, 07059 Antalya, Turkey, fax: +90 5304167543, e-mail: drahmetbasturk@hotmail.com

Pancreatitis is histologically defined as inflammation of the pancreatic parenchyma. Acute pancreatitis is a disorder manifested by interstitial oedema, acute decrease in inflammatory cells, apoptosis, necrosis, and reversible improvement in haemorrhage [1]. Azathioprine (AZA) is among the drugs associated with acute pancreatitis, although encountered rarely. Our objective is to present a paediatric case with ulcerative colitis (UC), who developed acute pancreatitis during AZA treatment, and was treated (after the discontinuation of azathioprine) with infliximab due to the relapse of UC.

A 17-year-old paediatric patient applied to us with complaints of bloody stool, diarrhoea, abdominal pain, and weight loss, which emerged in the previous 2 months. Medical and familial history was normal. In the physical examination, height was measured as $168 \mathrm{~cm}$ (10-25 p), body weight $49 \mathrm{~kg}(<3 \mathrm{p})$, no mouth ulcers were observed, and bilateral sensitivity in the abdomen and increased bowel sounds were recorded. No anal abscess, fistula, or fissure were observed during the anal examination. Laboratory analysis revealed: haemoglo-

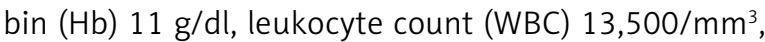
platelet count (PLT) $478,000 / \mathrm{mm}^{3}$, alanine aminotransferase (ALT) $28 \mathrm{U} / \mathrm{I}(\mathrm{N}: 0-40)$, aspartate aminotransferase (AST) $32 \mathrm{U} / \mathrm{l}(\mathrm{N}$ : 0-41), $\gamma$-glutamyl transferase (GGT) $24 \mathrm{U} / \mathrm{l}$ (N: 0-61), total bilirubin (T.bil.) $0.9 \mathrm{mg} / \mathrm{dl}$ (N: 0-0.9), direct bilirubin (D.bil.) $0.2 \mathrm{mg} / \mathrm{dl}(\mathrm{N}: 0-0.2)$, prothrombin time (PT) $14.6 \mathrm{~s}$ (N: 11-14), international normalised ratio (INR) 1.1 (N: 0.9-1.2), activated partial thromboplastin time (aPTT) $26.1 \mathrm{~s}$ (N: 25-33), albumin $4.2 \mathrm{~g} / \mathrm{dl}$, and faecal occult blood (HHb) 234. Stool culture was negative. Rotavirus, adenovirus and Entamoeba histolytica antigens were also negative. The upper endoscopy, which was carried out due to suspected inflammatory bowel disease, displayed antral gastritis and mild duodenitis, which was confirmed with histopathological analysis. In the colonoscopy, aphthous ulcerative areas were observed in the rectum, sigmoid colon, and caecum. The results of the multiple biopsies were interpreted in favour of ulcerative colitis. Methylprednisolone treatment, which was initiated with a loading dose of $2 \mathrm{mg} / \mathrm{kg}$ b.w./day, was planned for 6 weeks. Mesalazine $30 \mathrm{mg} / \mathrm{kg}$ b.w./day was concomitantly initiated. Azathioprine was started with a dose of $0.5 \mathrm{mg} / \mathrm{kg}$ b.w./day, and then the dose was increased to $2 \mathrm{mg} / \mathrm{kg}$ b.w./day. For the exacerbation periods, which occurred three times during the follow-up, methylprednisolone was administered. Towards the end of the 2-year follow-up, the patient was hospitalised duo to the severe abdominal pain radiating to the back. The patient had epigastric sensitivity during the physical examination. Laboratory analysis revealed: $\mathrm{Hb} 10 \mathrm{~g} /$

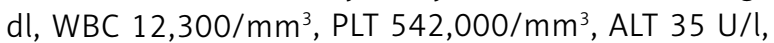
AST 36 U/l, GGT 28 U/l, T.bil. 0.8 mg/dl, D.bil. $0.1 \mathrm{mg} /$ dl, PT $11.3 \mathrm{~s}$, INR 1.0, aPTT $28.7 \mathrm{~s}$, albumin $3.9 \mathrm{~g} /$ dl, $\mathrm{HHb} 30$, pancreatic amylase $980 \mathrm{U} / \mathrm{l}$, and lipase $650 \mathrm{U} / \mathrm{l}$. The patient was diagnosed with acute pancreatitis after the findings in the ultrasound (US) were interpreted in favour of oedematous pancreatitis. Regarding the aetiology of pancreatitis, antinuclear antibodies (ANA), anti-smooth muscle antibody (ASMA), anti-neutrophil cytoplasmic antibody (ANCA) were not detected, and the viral serology was negative. Because the IgG levels were 980 ( $N$ : 600-1500) and lipid profile was normal, the patient was diagnosed with non-biliary pancreatitis. We believed that AZA was the aetiological cause of it, so azathioprine was discontinued.

Intravenous (IV) lansoprazole and parenteral nutrition were initiated and on the fourth day of follow-up, pancreatic amylase and lipase levels dropped to $40 \mathrm{U} / \mathrm{l}$ 
and $60 \mathrm{U} / \mathrm{l}$, respectively. The nutrition of the patient was gradually increased and was switched fully to the oral feeding. As the patient had a fifth exacerbation with bloody diarrhoea and abdominal pain during the follow-up; an exacerbation of the ulcerative colitis was suspected, and IV infliximab (5 $\mathrm{mg} / \mathrm{kg}$ b.w./day) was initiated in combination with methylprednisolone for 6 weeks. The patient received loading doses at the start and in the second and sixth weeks. Thereafter, management treatment was administered in every eighth week. Because we observed weight loss and diarrhoea in the $24^{\text {th }}$ week after the initiation of infliximab, we believed that we had a secondary nonresponding case. The patient was hospitalised and, as well as the routine examination, antigens for Clostridium difficile-PCR, CMV-PCR, and Entamoeba histolytica in stools were investigated because we suspected secondary colitis of the infectious origin. Because the $C$. difficile polymerase chain reaction test was positive, we initiated oral metronidazole (30 mg/kg b.w./day). But the treatment was changed to oral vancomycin ( $40 \mathrm{mg} / \mathrm{kg}$ b.w./day) when no response was received in the treatment, whereupon improvement was seen. The patient had no active complaints during the follow-up, and he was discharged. The patient is still under the treatment of infliximab, and he is followed-up in our outpatient department.

Although it does not have declared validity for the paediatric population, according to the Atlanta criteria, there should be two of the three following criteria for the diagnosis of acute pancreatitis: 1) clinical findings of pancreatitis, 2) amylase and lipase levels above three times of the normal levels, and 3) radiography in line with acute pancreatitis [1]. In our case, acute pancreatitis was diagnosed with clinical, laboratory, and ultrasound findings. Azathioprine is known as an affirmed cause of drug-induced pancreatitis in the transplantation and inflammatory bowel disease (IBD) patients [2]. In the animal experiments, it was biochemically and histologically shown that AZA induced pancreatitis due to the necrosis of the acinar cells caused by AZA [3]. Pancreatitis associated with AZA is dose-independent and usually has a mild course. However, pancreatic abscess might emerge and progress to complicated pancreatitis [4]. Pancreatitis in our case, regressed after the discontinuation of AZA and non-complication was observed. During AZA-induced pancreatitis, continuation of the AZA treatment is contraindicated. The average of the incidence of the AZA-induced pancreatitis is between $1.4 \%$ and $1.6 \%$, which means an eight-fold increased risk compared with the general population [5]. Tumour necrosis factor $\alpha$ (TNF- $\alpha$ ) is extensively expressed in the colon mucosa of patients with Crohn's disease (CD) and UC. It plays a major role in mucosal inflammation in
CD and UC patients, and excessive amounts are found in the stool, urine, and rectal secretions of patients with UC [6]. In several placebo-controlled studies and meta-analyses, it was observed that infliximab, which is a TNF- $\alpha$ inhibitor, is effective in adult patients diagnosed with UC $[7,8]$. Although its long-term risks in the paediatric population are not known, Miele et al. [9] reported that short-term treatment with infliximab was effective in 14 of 17 patients diagnosed with moderate to severe UC. In our case, infliximab treatment was initiated because UC had relapsed after the discontinuation of AZA. It was reported that $3 \%$ of the patients treated with infliximab developed severe infection [10]. It was also stated that infliximab treatment increases the risk of the opportunistic infection by up to three times. The C. difficile infection that was encountered in our case during the follow-up period improved with oral antibiotic therapy $[11,12]$.

In conclusion, regarding this case, it should be kept in mind that pancreatitis might develop with azathioprine, which is frequently used in the long-term management of UC. In addition, it also should be kept in mind that patients with recurrent exacerbations of UC might go into remission with the use of TNF- $\alpha$ inhibitors, but secondary infections (esp. C. difficile) might emerge in secondary non-responding patients.

\section{Conflict of interest}

The authors declare no conflict of interest.

\section{References}

1. Bradley EL 3rd. A clinically based classification system for acute pancreatitis. Summary of the International Symposium on Acute Pancreatitis, Atlanta, Ga, September 11 through 13, 1992. Arch Surg 1993; 128: 586-90.

2. Herrlinger KR, Stange EF. The pancreas and inflammatory bowel diseases. Int J Pancreatol 2000; 27: 171-9.

3. Foitzik T, Forgacs B, Ryschich E, et al. Effect of different immunosuppressive agents on acute pancreatitis: a comparative study in an improved animal model. Transplantation 1998; 65: 1030-6.

4. Haber CJ, Meltzer SJ, Present DH, et al. Nature and course of pancreatitis caused by 6-mercaptopurine in the treatment of inflammatory bowel disease. Gastroenterology 1986; 91: 982-6.

5. Bermejo F, Lopez-Sanroman A, Taxonera C, et al. Acute pancreatitis in inflammatory bowel disease, with special reference to azathioprine-induced pancreatitis. Aliment Pharmacol Ther 2008; 28: 623-8.

6. Saiki T, Mitsuyama K, Toyonaga A, et al. Detection of pro- and anti-inflammatory cytokines in stools of patients with inflammatory bowel disease. Scand I Gastroenterol 1998; 33: 616-22.

7. Ford AC, Sandborn WJ, Khan KJ, et al. Efficacy of biological therapies in inflammatory bowel disease: systematic review and meta-analysis. Am J Gastroenterol 2011; 106: 644-59. 
8. Probert CS, Hearing SD, Schreiber S, et al. Infliximab in moderately severe glucocorticoid resistant ulcerative colitis: a randomised controlled trial. Gut 2003; 52: 998-1002.

9. Miele E, Markowitz JE, Mamula P, et al. Human antichimeric antibody in children and young adults with inflammatory bowel disease receiving infliximab. J Pediatr Gastroenterol Nutr 2004; 38: 502-8.

10. Rutgeerts P, Sandborn WJ, Feagan BG, et al. Infliximab for induction and maintenance therapy for ulcerative colitis. N Engl J Med 2005; 353: 2462-76.

11. Domm S, Cinatl J, Mrowietz U. The impact of treatment with tumour necrosis factor-alpha antagonists on the course of chronic viral infections: a review of the literature. Br J Dermatol 2008; 159: 1217-28.

12. Hansen RA, Gartlehner G, Powell GE, et al. Serious adverse events with infliximab: analysis of spontaneously reported adverse events. Clin Gastroenterol Hepatol 2007; 5: 729-35.

Received: 18.04.2017

Accepted: 16.06.2017 